\title{
Assessing round window depiction in a virtual reality environment for cochlear implantation
}

\section{Justin T. Lui, ${ }^{a}, *$ Garrett D. Locketz, ${ }^{b}$ Joseph C. Dort, ${ }^{c}$ Joseph M. Chen, ${ }^{a}$ Justin K. Chau, ${ }^{c}$ Sonny K. Chan, ${ }^{c}$ Sumit K. Agrawal, ${ }^{d}$ J. Kenneth Salisbury ${ }^{e}$ and Nikolas H. Blevins ${ }^{f}$}

${ }^{a}$ Department of Otolaryngology-Head \& Neck Surgery, University of Toronto, Toronto, Ontario, M4N 3M5, Canada; ${ }^{b}$ Department of Otorhinolaryngology-Head \& Neck Surgery, University of Pennsylvania, Philadelphia, PA 19103, USA; ${ }^{c}$ Division of Otolaryngology-Head \& Neck Surgery, Department of Surgery, University of Calgary, Calgary, Alberta, T2T 5C7, Canada; ${ }^{d}$ Department of Otolaryngology, Western University, London Health Sciences Centre, University Hospital Otolaryngology, London, Ontario, N6A 5A5, Canada; ${ }^{e}$ Department of Computer Science, Stanford University, Stanford, CA 93405, USA; ${ }^{f}$ Department of Otolaryngology-Head \& Neck Surgery, Stanford University, Stanford, CA 94305, USA

${ }^{*}$ Corresponding author at: Department of Otolaryngology-Head \& Neck Surgery, University of Toronto, 2075 Bayview Avenue, Room M1 102, Toronto, Ontario, M4N 3M5, Canada.Email: jt.lui@mail.utoronto.ca

Date accepted for publication: 10 September 2020

\section{Abstract}

Background: A recently developed patient-specific virtual reality (VR) simulator has previously shown value in surgical rehearsal, but not clinical utility. Adequate round window exposure during cochlear implantation dictates the route of electrode insertion, however, anatomic variability among patients exists. Using patient-specific specimens, this study assessed the VR platform's ability to depict round window exposure during cochlear implantation surgery to evaluate its clinical utility. Methods: Retrospective data from 21 patients who had undergone cochlear implantation surgery by a single surgeon were collected. Based on the operative notes and video, round window exposure was classified as grade I $(\geqslant 50 \%)$ or grade II $(<50 \%)$. Segmented preoperative clinical computed tomography datasets were loaded into a custom surgical rehearsal platform. Six experienced cochlear implantation otologists (including the operative surgeon) from four institutions performed virtual cochlear implantation surgery and graded round window exposure. These results were compared with the intraoperative findings. Results: Overall, VR grading was congruent with the intraoperative gold standard in $78.6 \%$ (95\% confidence interval, $73.3 \%-83.8 \%$ ) of cases. Surgeons were more likely to correctly identify grade I (86.9\%) than grade II (61.9\%) exposures. The primary surgeon identified all five cases requiring a cochleostomy in comparison with the secondary surgeons, who correctly identified $48.0 \%$ (95\% confidence interval, $14.7 \%-81.3 \%$ ) of the cases. Conclusion: Surgically relevant temporal bone anatomic variations can be accurately identified with VR rehearsal. Accuracy may depend on individual surgical technique, because one surgeon's intraoperative findings may not be replicated by another's rehearsal. Further prospective assessments will help establish the utility of VR rehearsal in cochlear implantation preparation.

Keywords: virtual reality simulation; otology; temporal bone surgery; case-specific rehearsal; surgical rehearsal; surgical training

\section{Introduction}

Successful otologic surgery necessitates a strong understanding of the intricacies of temporal bone anatomy, which has traditionally been obtained from didactic teaching to handson exposure in the laboratory or operating room. ${ }^{1,2} \mathrm{~A}$ continued push for patient safety, improved clinical outcomes, and increasing surgical laboratory costs have resulted in the adoption of virtual reality (VR) simulation in the context of otolaryngology-head and neck surgery (OHNS) resident training. ${ }^{3-5}$

Virtual reality simulation has been explored in numerous surgical specialties, enabling the potential for limitless practice iterations in a safe and controlled environment. ${ }^{2,6,7}$

Presented as a poster at the American Otologic Society meeting at the Combined Otolaryngologic Spring Meeting, April 18-22, 2018, in National Harbor, MD, USA. 
Several computer-based VR simulation platforms exist for temporal bone surgery, and they vary in visual and tactile realism. ${ }^{8,9}$ VR simulation in temporal bone surgery has successfully demonstrated improved trainee performance in mastoidectomy performance after VR simulation; however, associated clinical significance has not been fully explored. ${ }^{1,10,11}$

Most simulators utilize standardized models and scenarios that may be encountered in the clinical setting. An alternative approach is patient-specific rehearsal, in which the user performs a virtual procedure on the upcoming patient's specific anatomy before the actual surgery to gain insight into the patient's anatomic differences or potential hazards.

CardinalSim, ${ }^{12}$ a patient-specific virtual surgical rehearsal platform, was developed via a multi-institutional effort. Several validation studies have assessed CardinalSim's potential utility. ${ }^{13-16}$ The system is capable of generating morphologically accurate three-dimensional renderings from clinical imaging data, such as computed tomography (CT) and/or magnetic resonance imaging. Users may then interact with these models to emulate the movement of a patient's head in the operating room while removing tissue as desired using high-fidelity haptic force feedback to produce an immersive experience for surgical planning and training. ${ }^{17}$

For a patient-specific rehearsal platform to be clinically useful, it must be capable of demonstrating anatomic variations and configurations that will affect the course of a procedure. In addition, the rehearsal should allow surgeons to anticipate challenges and guide preparation by accurately depicting anatomy and surgical constraints. The depiction of the round window in a facial recess approach of cochlear implantation is one such variable that has high anatomic variation among patients. In the setting of adequate round window exposure, a cochlear implant electrode can be inserted directly into the scala tympani, which has been shown to result in reduced cochlear trauma and improved hearing outcomes. Upon activation, the cochlear implant transmits externally processed sound into electrical impulses via the electrode to spiral ganglion cells, which helps restore hearing. ${ }^{18}$ However, if the round window is less than $50 \%$ visible during cochlear implantation, a cochleostomy may need to be performed for electrode insertion. ${ }^{19}$

Given the increasing number of cochlear implant recipients in the context of expanding clinical indications, there is a tremendous opportunity for VR surgical rehearsal to alter the current preoperative preparation paradigm. Replacing static review of clinical imaging with virtual surgical rehearsal would require improved simulation fidelity to accurately render microscopic structures. ${ }^{20}$ This objective measurement of anatomic variations in a virtual environment has not been well explored in temporal bone surgery. ${ }^{4}$ The present study sought to evaluate CardinalSim's clinical utility through its ability to depict the $2.5 \mathrm{~mm}^{2}$ round window via a facial recess approach to cochlear implantation.

\section{Materials and methods}

Retrospective surgical records of 21 patients who had undergone cochlear implantation surgery by a single surgeon at a single institution over the course of 6 months were selected to emulate seven operating lists. All procedures were undertaken with the intention of round window electrode insertion. One quarter of the cases with documented challenging anatomy necessitating cochleostomy were specifically included. Upon completion of the facial recess dissection and removal of the round window niche, round window exposure was classified as either grade I $(\geqslant 50 \%)$ or grade II $(<50 \%)$ by the operative surgeon (N.H.B.) at the time of surgery. All cases underwent complete electrode insertion. This was verified post-operatively by two authors (J.T.L. and G.D.L.) with operative video and surgical documentation.

Preoperative clinical imaging and associated radiology reports were reviewed by two authors (J.T.L. and G.D.L.) to ensure no abnormalities of the facial nerve, chorda tympani, and cochlea were present. The clinical CT datasets were stored in anonymized DICOM format (Digital Image Communication in Medicine) and loaded into Amira 5 (Zuse Institute Berlin, Germany) for segmentation of critical structures. Segmented structures included the facial nerve, chorda tympani, round window membrane and niche, sigmoid sinus, internal carotid artery, and ossicular chain. Segmentation was performed by a digital artist with previous experience in generating a library of virtual specimens for postgraduate training. Virtual three-dimensional models were then generated from these segmented datasets in CardinalSim and anatomic accuracy was determined by two authors (J.T.L. and N.H.B.). Original DICOM data were displayed as a volumetric representation of bone using a pre-set threshold value of 450 Hounsfield units. The creation of virtual models required approximately 45 minutes for each dataset.

Utilizing consumer-grade computing hardware, CardinalSim is compatible with Windows (Microsoft, Redmond, WA), Linux and Mac (Apple, Cupertino, CA) operating systems. In this investigation, a nVIDIA (Santa Clara, CA) GeForce GTX 970 4GB graphics card coupled with an Intel (Santa Clara, CA) Core i7 $3.6 \mathrm{GHz}$ processor and $16 \mathrm{~GB}$ of DDR3 
RAM was used. The display units used included passive LG (Seoul, South Korea) 3D monitors or Sony (Tokyo, Japan) PlayStation 3D Displays with SimulView technology. All centres used 3D Systems (Valencia, CA) Geomagic Touch haptic devices.

Five fellowship-trained otologists specializing in cochlear implantation in their respective centres from four institutions were provided with a brief instruction guide on CardinalSim and the haptic device. Several examples of round window exposures were shown to the surgeons during this orientation to demonstrate grade I and II differentiation. In randomized order, these five secondary surgeons in addition to the primary surgeon were asked to perform a virtual mastoidectomy and facial recess dissection as they would during actual surgery. They were asked to preserve the facial nerve, chorda tympani, posterior canal wall and bony annulus. ${ }^{19}$ The round window niche was removed when necessary to facilitate round window exposure. After each virtual dissection, each otologist assigned each dataset a round window binary grading based on the percentage of visualization (grade $\mathrm{I} \geqslant 50 \%$ or grade II $<50 \%$ ). The surgeons were blinded to patient data including intraoperative findings.

Grades from actual and virtual dissections were compared. Subset analysis was also performed on the primary surgeon's ability to accurately identify challenging grade II round window exposures that required cochleostomy based on virtual dissections. Inter-rater reliability was calculated using Cohen's kappa analysis, which compared the agreement between surgeons' round window grades. CT quality and other characteristics were explored to identify modifiable factors that contributed to variability. Data analysis was completed using SPSS version 24 (IBM, Armonk, NY) and Microsoft Excel (Redmond, WA). The host Institutional Review Board approval was granted for this study (IRB-6208).

\section{Results}

Of the 21 cochlear implantation cases, there were 11 right and 10 left ears (10 female, 11 male). The mean age of the patients was 56.4 years including two paediatric patients aged 6 and 13 years (Table 1). In addition, CT scans were direct axial plane images of the temporal bone using a helical technique (slice thickness, $0.625 \mathrm{~mm}$; matrix, $512 \times 512$, $120 \mathrm{kV}, 350 \mathrm{~mA}$ ). Fourteen cases were classified as grade I, and seven were classified as grade II round window exposure. Virtual and intraoperative facial recess dissections of the primary surgeon are depicted in Fig. 2.

The overall accuracy of the primary operative surgeon to identify the correct round window exposure was $81 \%$, and the secondary surgeons scored $78.1 \%$ (95\% confidence
Table 1. Patient demographics and intraoperative findings

\begin{tabular}{|c|c|c|c|c|}
\hline $\begin{array}{l}\text { Patient } \\
\text { no. }\end{array}$ & $\begin{array}{l}\text { Age } \\
\text { (years) }\end{array}$ & $\begin{array}{l}\text { Cochlear } \\
\text { implant } \\
\text { insertion } \\
\text { side }\end{array}$ & $\begin{array}{l}\text { Gold } \\
\text { standard } \\
\text { round window } \\
\text { grading }\end{array}$ & Cochleostomy \\
\hline 1 & 45 & Right & 1 & \\
\hline 2 & 76 & Left & 2 & Yes \\
\hline 3 & 86 & Right & 1 & \\
\hline 4 & 47 & Left & 2 & Yes \\
\hline 5 & 59 & Left & 2 & Yes \\
\hline 6 & 48 & Right & 1 & \\
\hline 7 & 70 & Right & 2 & Yes \\
\hline 8 & 25 & Left & 2 & \\
\hline 9 & 13 & Right & 1 & \\
\hline 10 & 70 & Right & 1 & \\
\hline 11 & 68 & Right & 1 & \\
\hline 12 & 77 & Left & 1 & \\
\hline 13 & 86 & Left & 1 & \\
\hline 14 & 6 & Right & 1 & \\
\hline 15 & 53 & Left & 1 & \\
\hline 16 & 47 & Left & 1 & \\
\hline 17 & 78 & Left & 2 & Yes \\
\hline 18 & 83 & Right & 1 & \\
\hline 19 & 80 & Right & 2 & \\
\hline 20 & 23 & Left & 1 & \\
\hline 21 & 44 & Right & 1 & \\
\hline
\end{tabular}

interval [CI], 71.4\%-84.6\%). When combining all surgeons' scores, grade I and II exposures were correctly identified in 86.9\% (95\% CI, 75.9\%-98.0\%) and 61.9\% (95\% CI, 30.9\%$92.9 \%$ ) of cases, respectively. With respect to grade I ( $\geqslant 50 \%$ round window visibility) cases, the primary surgeon correctly identified $71.4 \%$ of exposures and the secondary surgeons scored $90.0 \%$ (95\% CI, $79.9 \%-100.0 \%)$ of the exposures correctly (Table 2$)$. For grade II $(<50 \%$ round window visibility) cases, the primary surgeon correctly graded $100.0 \%$ of exposures, whereas only $54.3 \%$ (95\% CI, $22.6 \%-86.0 \%$ ) of these cases were accurately identified by the secondary surgeons.

Of the seven grade II cases, five patients required a cochleostomy for electrode placement. A cochleostomy identification score was calculated to determine the percentage of cases requiring cochleostomy that were appropriately identified (Table 1). For the primary surgeon, $100 \%$ of the cochleostomy cases were identified in contrast to $48.0 \%$ (95\% CI, 14.7\%-81.3\%) for the secondary surgeons.

For all cases and all surgeons, the intraclass correlation coefficient (ICC) was $0.64(0.36-0.83)$ when a two-way mixed effects model using absolute agreement and multiple raters 
Table 2. Surgeon scoring when comparing virtual reality round window exposure with intraoperative gold standard of all 21 cases

\begin{tabular}{|c|c|c|c|c|c|c|c|c|}
\hline & \multirow{2}{*}{$\begin{array}{l}\text { Primary } \\
\text { surgeon (\%) }\end{array}$} & \multicolumn{6}{|c|}{ Secondary surgeons (\%) } & \multirow{2}{*}{$\begin{array}{l}\text { All surgeons } \\
(\%)\end{array}$} \\
\hline & & Surgeon 2 & Surgeon 3 & Surgeon 4 & Surgeon 5 & Surgeon 6 & Average & \\
\hline Grade I accuracy ( $\geqslant 50 \%$ round window) & 71.4 & 92.9 & 85.7 & 92.9 & 100.0 & 78.6 & $90.0 \pm 8.1$ & $86.9 \pm 10.5$ \\
\hline Grade II accuracy ( $<50 \%$ round window) & 100.0 & 28.6 & 57.1 & 71.4 & 28.6 & 85.7 & $54.3 \pm 25.5$ & $61.9 \pm 29.5$ \\
\hline Overall accuracy & 81.0 & 71.4 & 76.2 & 85.7 & 76.2 & 81.0 & $78.1 \pm 5.4$ & $78.6 \pm 5.0$ \\
\hline Cochleostomy identification score & 100.0 & 20.0 & 60.0 & 60.0 & 20.0 & 80.0 & $48.0 \pm 2.7$ & $56.7 \pm 32.0$ \\
\hline
\end{tabular}

The cochleostomy identification score assessed a surgeon's ability to identify which cases required a cochleostomy given the limited round window exposure.

was used. This was consistent with moderate to good reliability. ${ }^{21}$ Ten cases demonstrated complete surgeon consensus and agreement with the intraoperative gold standard. Of these cases, $91.0 \%$ had greater than $50 \%$ round window visualization. In contrast, the remaining 11 cases of the group lacking total consensus had a significantly reduced overall score of $55.0 \%$ (95\% CI, $40.0 \%-70.0 \%)$ and an ICC of $0.20(-0.93$ to 0.77$)$. Most cases $(85.7 \%)$ with intraoperative grade II round window exposures were within this group.

\section{Discussion}

Bordered by the chorda tympani, facial nerve and the incudal buttress, the facial recess is the most common path used for cochlear implantation electrode array insertion into the scala tympani (Fig. 1). Despite the performance of an optimal dissection, including the removal of the round window niche, a cochleostomy may be required if the $2.5 \mathrm{~mm}^{2}$ round window membrane is not visible. ${ }^{19,22}$ Drilling a cochleostomy enables direct electrode insertion into the scala tympani. In a case series of 128 consecutive cochlear implantations, less than 50\% round window visibility required at least an extended round window approach anteroinferiorly and/or cochleostomy. ${ }^{19}$ The frequency with which this is required intraoperatively is highly dependent on the surgeon's judgement and comfort with different techniques.

VR simulators have been shown to assist in the acquisition of surgical skill and knowledge in temporal bone surgery. ${ }^{4}$ Although its application in preoperative rehearsal has been explored, the impact has not been extensively assessed. ${ }^{4,13,23}$ Temporal bone simulation has traditionally lacked the capability to generate patient-specific virtual models from clinical diagnostic imaging, and thus has been limited to presenting standardized virtual specimens. Moreover, the transition from raw image files to segmented virtual specimens is far from a seamless, automated process. ${ }^{23}$

CardinalSim, a patient-specific VR surgical simulator, integrates clinical diagnostic imaging and has demonstrated

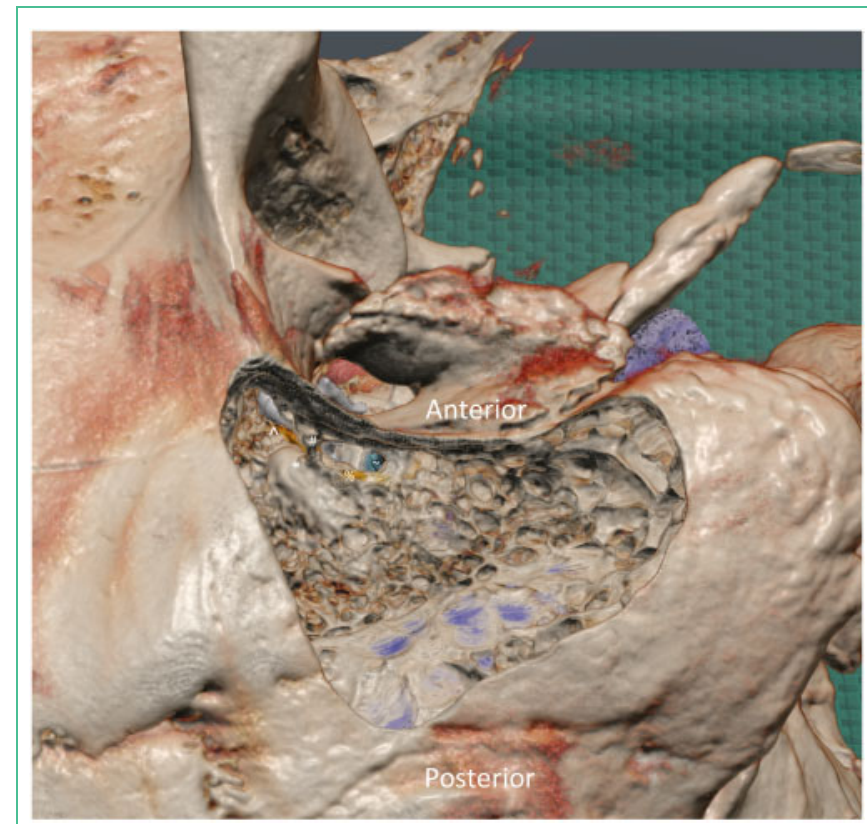

Figure 1. CardinalSim rendering of a dissected cortical mastoidectomy and facial recess dissection. The horizontal semicircular canal $(*)$ and the short process of the incus $\left({ }^{\wedge}\right)$ are used to determine the position of the facial nerve (vertical/mastoid segment). The facial recess is demarcated by the facial nerve (\%), chorda tympani, and the incus buttress (\#). The round window membrane $(\sim)$ is visible with the niche having been drilled away.

both face and construct validities. Its ability to accurately depict surgically relevant anatomic variations, however, has not been studied. ${ }^{4,13,17}$ Such capacity is critical in determining its potential as a preoperative rehearsal tool. Round window exposure through the facial recess is a prime opportunity to explore this possibility due to its proximity to nearby critical anatomy and high anatomic variation.

The overall accuracy of round window exposure after VR rehearsal in the present study of $78.6 \%$ (95\% CI, $73.3 \%-$ $83.8 \%)$ is encouraging. CardinalSim's virtual environment is therefore capable of providing experienced otologic surgeons with surgically meaningful information (Table 1). In addition, surgeons were better at identifying grade I exposures $(84.3 \%)$ than the more challenging grade II exposures (68.6\%). Paralleling the poor grade II accuracy was a 




Figure 2. Matched virtual and intraoperative facial recess dissections with (A) grade I ( $\geqslant 50 \%$ RW exposure) and (B) grade II $(<50 \%$ RW exposure).

cochleostomy identification score of only $56.7 \%$ for the secondary surgeons, which contrasted with the primary surgeon's $100.0 \%$ cochleostomy identification. In other words, secondary surgeons were more likely to regard grade II exposures as grade I in the virtual stetting. This discrepancy may be attributable to more aggressive virtual dissections of the secondary surgeons in the absence of risk to the facial nerve. In the operating room, surgeons may be more reluctant to expose significant portions of the facial nerve, which influences the decision to perform a cochleostomy.

In addition, factors that prevent additional dissection in the operating room may not be represented in VR, such as bone dust debris or a patient's limited neck rotation. As a result, different surgical techniques may lend themselves to different exposures between surgeons; the primary surgeon's virtual dissection may be more consistent with their techniques used intraoperatively. Therefore, using the operative surgeon as the gold standard may not accurately reflect the intraoperative findings of other surgeons. This challenges the strength of the accuracy scoring of our secondary surgeons, especially in the setting of a limited number of surgeons. To overcome this, a design that pairs surgeons' VR findings with their own intraoperative findings should be performed. If VR dissections were performed prospectively and were surgeon specific, it may equip surgeons with insight on the trajectory of dissection, the amount of facial recess drilling required, or even the posterior extent of the cortical mastoidectomy.

Although the simulation aspect was performed prospectively, one major limitation was the retrospective collection of operative cases. We attempted to avoid recall bias of the primary surgeon by having two other author (J.T.L. and G.D.L.) generate anonymized virtual specimens, which were presented in a blinded, randomized order. Moreover, surgeon familiarity and technological understanding of
CardinalSim was variable and the skill disparity potentially affected virtual dissections. This may have manifested in different fields of view obtained by each surgeon, resulting in more or less bone removal. As a result, the round window exposure may have been affected.

Another potential limitation includes the intentional selection of difficult grade II round window exposures, which is considerably higher than would be expected in clinical practice. The non-operative participating surgeons were not made aware of the increased representation of challenging cases, which may have created the expectations that fewer grade II datasets would be encountered during virtual dissection.

In this investigation, our goal was to highlight the use of patient-specific VR surgeries in depicting microscopic anatomy in temporal bone surgery, which had not been previously performed. An acceptable level of accuracy between intraoperative and VR findings for both primary and secondary surgeons was discovered. However, the large discrepancy within cochleostomy identification is highly suggestive of variability among surgeons' technique and performance. Further prospective and surgeon-case matched analysis should be pursued to further elucidate the nuances of patient-specific VR rehearsal. This study highlights opportunities for improvement of CardinalSim, which is undergoing iterative upgrades to graphics and features, such as the addition of auto-segmentation of critical anatomy and user performance feedback.

\section{Conclusion}

The goal of virtual rehearsal is to guide a surgeon when undertaking a specific procedure, using familiar techniques 
while practicing consistent caution. By depicting the complex anatomy of the facial recess and the round window, it was demonstrated that patient-specific VR simulation is a promising modality for preoperative preparation in temporal bone surgery. By combining realistic visual renderings and accurate haptic feedback based on clinical imaging, virtual rehearsal allows surgeons to prepare for challenging anatomic configurations before performing surgery on a patient. The transference of skills from surgical simulation to the operating room remains unknown. Prospective studies may benefit from having surgeons act as their own comparison.

\section{Acknowledgements}

The authors would like to thank Laura Siegel for her contributions to language editing. The funding for this project stems from the National Institutes of Health (NIH) grant R01 LM010673-01A1.

\section{Conflict of interest}

None declared

\section{References}

1. Francis HW, Malik MU, Diaz Voss Varela DA, Barffour MA, Chien WW, Carey JP, et al. Technical skills improve after practice on virtual-reality temporal bone simulator. Laryngoscope 2012; 122: 1385-1391. https://doi.org/10.1002/ lary.22378.

2. Khemani S, Arora A, Singh A, Tolley N, Darzi A. Objective skills assessment and construct validation of a virtual reality temporal bone simulator. Otol Neurotol 2012; 33: 1225-1231. https://doi.org/10.1097/MAO.0b013e31825e7977.

3. Butler NN, Wiet GJ. Reliability of the Welling scale (WS1) for rating temporal bone dissection performance. Laryngoscope 2007; 117: 1803-1808. https://doi.org/10.1097/MLG.0b013e3 1811edd7a.

4. Lui JT, Hoy MY. Evaluating the effect of virtual reality temporal bone simulation on mastoidectomy performance: a meta-analysis. Otolaryngol Head Neck Surg 2017; 156: 10181024. https://doi.org/10.1177/0194599817698440.

5. Lui JT, Compton ED, Ryu WHA, Hoy MY. Assessing the role of virtual reality training in Canadian Otolaryngology-Head \& Neck Residency Programs: a national survey of program directors and residents. J Otolaryngol Head Neck Surg 2018; 47: 61. https://doi.org/10.1186/s40463-018-0309-4.

6. Aggarwal R, Black SA, Hance JR, Darzi A, Cheshire NJW. Virtual reality simulation training can improve inexperienced surgeons' endovascular skills. Eur J Vasc Endovasc Surg 2006; 31: 588-593. https://doi.org/10.1016/j.ejvs.2005.11.009.
7. Woodrum DT, Andreatta PB, Yellamanchilli RK, Feryus L, Gauger PG, Minter RM. Construct validity of the LapSim laparoscopic surgical simulator. Am J Surg 2006; 191: 28-32. https://doi.org/10.1016/j.amjsurg.2005.10.018.

8. Kashikar TS, Kerwin TF, Moberly AC, Wiet GJ. A review of simulation applications in temporal bone surgery. Laryngoscope Investig Otolaryngol 2019; 4: 420-424. https:// doi.org/10.1002/lio2.277.

9. Bhutta MF. A review of simulation platforms in surgery of the temporal bone. Clin Otolaryngol 2016; 41: 539-545. https:// doi.org/10.1111/coa.12560.

10. Sethia R, Wiet GJ. Preoperative preparation for otologic surgery: temporal bone simulation. Curr Opin Otolaryngol Head Neck Surg 2015; 23: 355-359. https://doi.org/10.1097/MOO. 0000000000000181.

11. O'Leary SJ, Hutchins MA, Stevenson DR, Gunn C, Krumpholz A, Kennedy G, et al. Validation of a networked virtual reality simulation of temporal bone surgery. Laryngoscope 2008; 118: 1040-1046. https://doi.org/10. 1097/MLG.0b013e3181671b15.

12. CardinalSim [Internet]. Available from: https://cardinalsim. stanford.edu/.

13. Locketz GD, Lui JT, Chan S, Salisbury K, Dort JC, Youngblood P, et al. Anatomy-specific virtual reality simulation in temporal bone dissection: perceived utility and impact on surgeon confidence. Otolaryngol Head Neck Surg 2017; 156: 1142-1149. https://doi.org/10.1177/0194599817691474.

14. Chan S, Li P, Lee DH, Salisbury JK, Blevins NH. A virtual surgical environment for rehearsal of tympanomastoidectomy. Stud Health Technol Inform 2011; 163: 112-118. https://doi. org/10.3233/978-1-60750-706-2-112.

15. Won T-B, Hwang P, Lim JH, Cho S-W, Paek SH, Losorelli S, et al. Early experience with a patient-specific virtual surgical simulation for rehearsal of endoscopic skull-base surgery. Int Forum Allergy Rhinol 2018; 8: 54-63. https://doi.org/10. 1002/alr.22037.

16. Compton EC, Agrawal SK, Ladak HM, Chan S, Hoy M, Nakoneshny SC, et al. Assessment of a virtual reality temporal bone surgical simulator: a national face and content validity study. J Otolaryngol Head Neck Surg 2020; 49: 17. https://doi. org/10.1186/s40463-020-00416-7.

17. Chan S, Li P, Locketz G, Salisbury K, Blevins NH. High-fidelity haptic and visual rendering for patient-specific simulation of temporal bone surgery. Comput Assist Surg 2016; 21: 85101. https://doi.org/10.1080/24699322.2016.1189966.

18. Stakhovskaya O, Sridhar D, Bonham BH, Leake PA. Frequency map for the human cochlear spiral ganglion: implications for cochlear implants. J Assoc Res Otolaryngol 2007; 8: 220-233. https://doi.org/10.1007/s10162-007-0076-9.

19. Leong AC, Jiang D, Agger A, Fitzgerald-O'Connor A. Evaluation of round window accessibility to cochlear implant 
insertion. Eur Arch Otorhinolaryngol 2013; 270: 1237-1242. https://doi.org/10.1007/s00405-012-2106-4.

20. Health Quality Ontario. Bilateral cochlear implantation: a health technology assessment. Ont Health Technol Assess Ser 2018; 18(6): 1-139. PMID: 30443278.

21. Koo TK, Li MY. A guideline of selecting and reporting intraclass correlation coefficients for reliability research. J Chiropr Med 2016; 15: 155-163. https://doi.org/10.1016/j.jcm.2016.02. 012.
22. Su W-Y, Marion MS, Matz GJ, Hinojosa R. Anatomical measurements of the cochlear aqueduct, round window membrane, round window niche, and facial recess. Laryngoscope 1982; 92: 483-486. https://doi.org/10.1288/00005537-198205 000-00003.

23. Arora A, Swords C, Khemani S, Awad Z, Darzi A, Singh A, et al. Virtual reality case-specific rehearsal in temporal bone surgery: a preliminary evaluation. Int J Surg 2014; 12: 141145. https://doi.org/10.1016/j.ijsu.2013.11.019. 